\title{
Texture in airborne SAR imagery of tropical forest and its relationship
} to forest regeneration stage

\author{
A. J. LUCKMAN \\ Remote Sensing Applications Development Unit, British National Space \\ Centre, ITE Monks Wood, Abbots Ripton, Cambridgeshire, PE172LS, \\ England, U.K.
}

\section{A. C. FRERY}

Departmento de Informatica, Universidade Federal de Pernambuco, Caixa Postal 7851. 50732-970 Recife, PE, Brazil

\section{C. F. YANASSE}

Divisão de Processamento de Imagens, Instituto Nacional de Pesquisas Espaciais, Avenida dos Astronautas 1758, 12227-010 São José dos Campos, SP, Brazil

\section{and G. B. GROOM}

Department of Landscape Ecology, National Environmental Research Institute, Kaloe, Grenaavej 14. DK-8410 Roende, Denmark

\section{(Received 28 August 1995: in final form 21 August 1996)}

Abstract. At C-band, SAR imagery often exhibits little variation in mean amplitude between different types of natural land cover. However, there is frequently a large amount of information to be found in the textural properties of such imagery, especially when it is acquired at high spatial resolution. This textural information may be useful in observing processes that affect the homogeneity of land surface vegetation such as the staged succession of regenerating tropical forest following human disturbance which is characterized by the gradual decrease in canopy homogeneity as regrowth species are succeeded by hardwood species.

In this study, three techniques of measuring the texture in C-band airborne SAR imagery from a tropical forest region in central Brazil are compared. The dependence of these measures on the stage of forest regeneration is assessed by using a temporal sequence of Landsat TM imagery to independently estimate the age of regrowth. Each texture measure is able to discriminate well between mature forest and other classes of vegetation where the image amplitude alone cannot be used to make the same distinction. Further discrimination between different ages of regrowth appears possible but is difficult to demonstrate quantitatively because a satisfactory validation of regeneration age is difficult to achieve.

\section{Introduction}

The South American Radar EXperiment (SAREX) of May 1992 was designed to evaluate the usefulness of airborne and simulated spaceborne C-band Synthetic Aperture Radar (SAR) data for monitoring the tropical forest environment. When the Canada Centre for Remote Sensing (CCRS) Convair 580 SAR imagery from this campaign was delivered, initial evaluations suggested that the majority of information about the land surface cover within these tropical forest regions was carried in the

$$
\text { 0143-1161:97 \$12.01) ¿ } 1997 \text { Taylor \& Francis Ltd }
$$


image texture rather than in th: mage amplitude (Groom at al. 1993, Yanasse ot al. 1993. van der Sanden and Hoesinan 19931. This can be allributed to two factors:

(1) At C-band (:a wavelen $5:$ i of $5.66 \mathrm{~cm}$ ) the microwave radiation does not penetrate far into a regs:ation canopy and the backscaltering coefficient for mature forest is similar $: 2$ that for regenerating forest, agricultural crops, or even moist bare soil (G::ver and Quegan. 1995).

(2) The variation in canop: structure and canopy surficce roughness associated with the development of : egetation from clear-cut hrough stages of regeneration to mature scconda $\because \because$ forest occurs at a spaltial scale detectable at the high resolution $(6 \mathrm{~m})$ of the CCRS SAR.

The ability to discriminate $\mathrm{t}$ : ween mature forest. diflicrent stages of regenerating forest and pasture by measuris $z$ the variations in imagc lexture will support the remote observation of the tropic: forest biome. The stige of tropical forest succession following disturbance has an :afluence on many important factors such as the diversity of species. the local hy Erology and the rate of biomalss accumulation (Uhl 1987). The significanse of thes : actors to biodiversity and to the regional hydrological and carbon coles mak $=s$ the assessment of the extent and developmental stage of regenerating forest are $:$ s an important objective.

This paper examines three -.:ethods of quantifying the lexture within imagery, acquired during the SAREX cimpaign. of tropical forest al Tapajós in Brazil and investigates their relationship ti : $:$ generation age as derived llirough a time sequence of optical images.

\section{Test site and SAR image pri-processing}

The SAREX campaign acqt.:-ed airborne SAR data from six South and Central American countries containing : ige amounts of tropical forcst. This study examines the data from the Tapajos regita of central Amazonia in Britzil which was chosen for the variety of land use and :-se arailability of other radlitr datasets. The test site lies on the BR163 highway sout: of the Amazon port of Santarem and its location is shown in figure 1 . The $-4 \mathrm{~km}$. $24 \mathrm{~km}$ area of interest covers farms with pasture and cultivation. areas of regene:zting forest. and parts of the Tapajós forest reserve to the west of the highway.

During the SAREX campai - the CCRS Convair 580 airhorne SAR system was deployed from an altitude of $6 \mathrm{~km}$ at two wavelengths $(3.24 \mathrm{~cm}$ : X-band and $5.66 \mathrm{~cm}$ : C-band) in various polarizatior. :ombinations (although none fully polarimetric) and three imaging modes (wide swe:c. narrow swath and nadir mode) (Wooding et al. 1994). The C-band narrow swath data were used in this study because they exhibit the most distinctive texture varis:ions across the target. These data are dual polarization (HH and VV). --look. S-bit implitude imagery with a pixcl size of approximately $4 \mathrm{~m}$ and nominal resolution of $: \mathrm{m}$ in both the range and arimuth directions across an $18 \mathrm{~km}$ swath and with a rar.:tion in look angle between $45^{\circ}$ and $76^{\circ}$.

As the analysis of texture ises not rely on calibrated ditla and the calibration information supplied with the :-nagery proved to give unsaltisfactory results, a full calibration was not performed. Woodins et al. 1994. Hawvkins and Teany 1993). However. the azimuth stripin removed by finding the mean viue for each image column in the azimuth direction and using a fitted poly nomial fu-ction to remove the systemat tic across-swath pattern. This method satisfactorily remo:ed the azimuth striping while retaining all image 


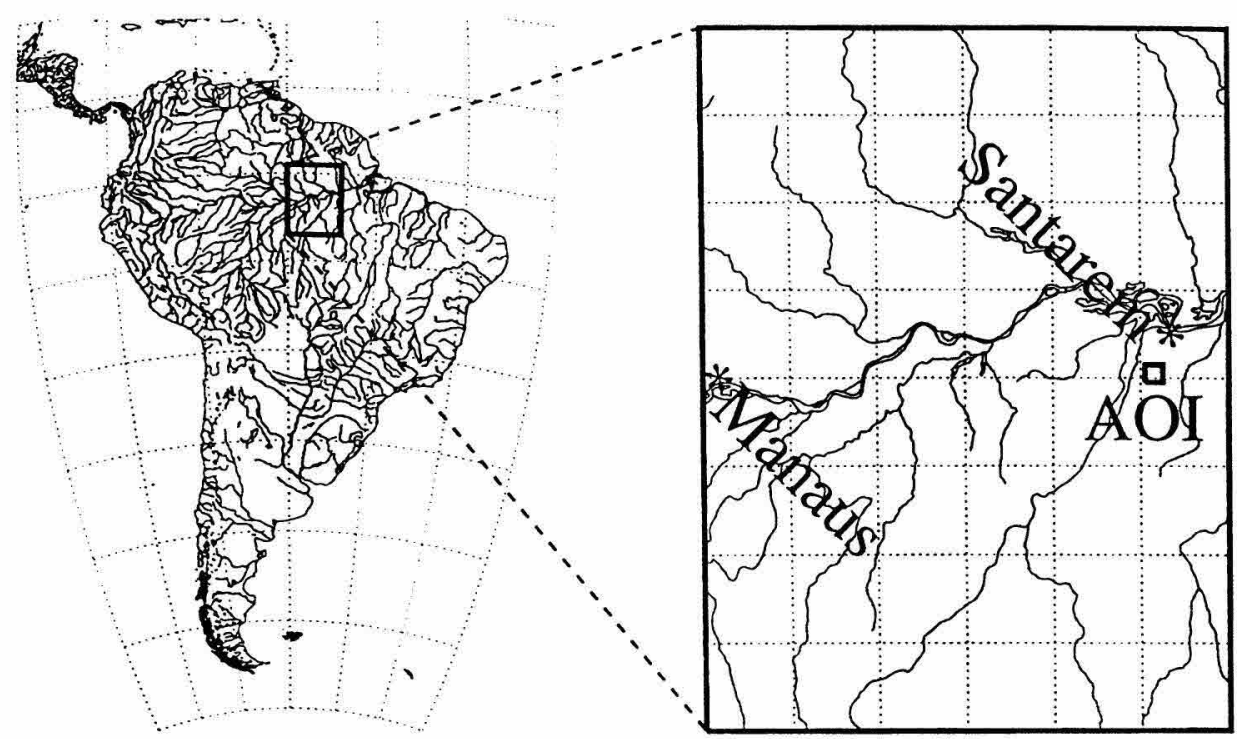

Figure 1. The location of the Tapajós test site within Brazil. The central Amazon region from Manaus to Santarém is shown in detail on the right. The box below Santarem shows the specific area of interest for this study.

textural variations and, although absolute comparisons between $\mathrm{HH}$ and VV were not subsequently valid, relative comparisons were still possible.

To allow a comparison of the SAR imagery with other image data, it was necessary to resample the imagery to a UTM projection. Any such resampling may be expected to affect the image statistics, and hence the image texture, especially where the spatial scale of the texture is similar to the pixel spacing (Quegan 1990). However, in this case, the spatial scale of the observed texture covered many pixels and the textural variations in the image were not appreciably affected by resampling. The resampling was performed by converting the $\mathrm{HH}$ and VV images from slant to ground range assuming an altitude of $6 \mathrm{~km}$. and co-registering them to a geocoded Landsat TM image (UTM projection). A nearest-neighbour resampling scheme and 24 tie-points were used to give a residual mean registration error of approximately $15 \mathrm{~m}$.

The resulting database contained two SAR images ( $\mathrm{HH}$ and VV polarizations) and represented an area of $24 \mathrm{~km}$ by $24 \mathrm{~km}$ with a pixel size of $6 \mathrm{~m}$ by $6 \mathrm{~m}$ making 4000 by 4000 pixels in total. The top-left UTM coordinate of this area is 726000 , 9686000 .

\section{Statistical image modelling}

\subsection{Background}

Image texture is characterized as the spatial variability in image tone and arises from the heterogeneity in target properties giving rise to different neighbouring pixel values for the same apparent target type. This spatial variability can be measured over a specific pixel distance, or within a given image area, and will be influenced by the underlying speckle arising from the nature of SAR imaging.

This study investigates three techniques for measuring texture; one based on an 
assumption of the statistical distributions underlying SAR imaging, one based on the first-order statistics of image areas and one second-order measure based on differences in pixel values over a specified distance.

The first of these techniques derives from an understanding of observations acquired under coherent illumination known as the multiplicative model (Oliver 1991). According to this model, a SAR observation $(z)$ can be considered as the product of the signal due to the backscattering coefficient of the target $(x)$ and the speckle noise associated with coherent imaging $(y)$. The distribution of the observations $(Z)$ at a certain position is that of the product of two independent random variables, one for the speckle noise $(Y)$ and one for the terrain backscatter $(X)$. The distributions of these random variables are considered for the amplitude imaging case.

\subsection{Speckle}

The physics of coherent illumination allow the assumption that a good model for the distribution of multilook amplitude speckle is given by the Square Root of Gamma distribution (Yanasse e't al. 1995). This is denoted as $\sqrt{\Gamma}(n, n)$ and characterized by the density function:

$$
f_{Y}(y ; n)=\frac{2 n^{n}}{\Gamma(n)} y^{2 n-1} e^{-n y^{2}} ; y, n>0
$$

where the parameter $n$ is a positive real value known as the equivalent number of looks.

Equation (1) is the density of the square root of the mean of $n$ intensity independent looks, since they all are exponentially distributed with unitary mean (fully developed speckle is considered here). The SAREX data is formed by taking the mean of amplitude looks and, since an explicit density for the arising distribution is not available for $n>2$, its distribution is approximated by the Square Root of Gamma.

In practice, the equivalent number of looks is smaller in general than the nominal number of looks because of the lack of complete stochastic independence between observations. This value can be measured as a function of the ratio of the standard deviation to the mean for image areas that exhibit no texture at the scale of the observation. In practice this means over smooth targets such as, in the case of Tapajós, over a region known to be homogeneous pasture.

\subsection{Distributions for the observed data}

For an entirely homogeneous target, the backscattering coefficient will be constant for neighbouring pixels. For a heterogeneous target, i.e., one exhibiting texture at the scale of observation, an approach based on empirical evidence and theoretical argument (Caves 1993) suggests that the distribution obeys a $\sqrt{\Gamma}(\alpha, \lambda)$ distribution with density function:

$$
f_{X}(x ; \alpha, \lambda)=\frac{2 i^{x}}{\Gamma(x)} x^{2 x-1} e^{-\lambda x^{2}} ; x, \alpha, \lambda>0
$$

The moments of this distribution are given by:

$$
E\left(Z^{r}\right)=\frac{\Gamma(x+r / 2)}{i^{r} \Gamma(\alpha)}
$$

One important property of this distribution is that its characteristic function converges to a constant. 
i.e.

$$
\text { if } x \rightarrow \propto \text { and } \lambda \rightarrow \propto \text {, such that } \alpha / \lambda \rightarrow \beta \in \mathcal{M}_{+}
$$

then

$$
X \sim \sqrt{\Gamma}(x . i) \stackrel{\operatorname{Pr}}{\longrightarrow} \beta
$$

Where $\stackrel{\mathrm{Pr}}{\longrightarrow}$ denotes convergence in probability. In other words, the variability of the random variable $X$ that obeys the $\sqrt{\Gamma}(\alpha, \lambda)$ law vanishes around the value $\alpha / \lambda$ as these parameters grow to infinity, if their ratio is finite.

Hence it can describe the distribution for textureless as well as textured targets, since the former are defined as those with constant backscatter and the latter are associated with varying backscatter.

\subsection{Combined statistics for observed pixel values}

According to the multiplicative model, if the speckle and backscatter are distributed according to equations (1) and (2) then the distribution of the random variable $Z=X Y$ is known as a multilook Amplitude $K$ distribution and is characterized by the density function

$$
f_{Z}(z ; \alpha, \beta, n)=\frac{4 z \alpha n}{\Gamma(\alpha) \beta \Gamma(n)}\left(\frac{\alpha n z^{2}}{\beta}\right)^{x+n-2 / 2} K_{x-n}\left(2 z \sqrt{\frac{\alpha n}{\beta}}\right) ; z, \alpha, \beta, n>0
$$

where $K_{r}$ is the modified $V$-order Bessel function of third kind and $\beta=\alpha / i$ and $\beta$ is the second order moment of the random variable :.

This distribution is denoted $K A(\alpha, \beta, n)$ and its moments are given by

$$
E\left(Z^{r}\right)=\left(\frac{\beta}{\alpha n}\right)^{r / 2} \frac{\Gamma(r / 2+n) \Gamma(r / 2+\alpha)}{\Gamma(\alpha) \Gamma(n)}
$$

Some properties of this distribution can be used to retrieve textural information from SAR image data.

\section{Texture measures}

4.1. K-distribution $\propto$ parameter

The degree of texture or inhomogeneity within the target can be assessed by examining the distribution of the population of pixels from that target. If they are $\sqrt{\Gamma}(n, n / \beta)$ distributed then the target has little or no texture. If they are $K A(\alpha, \beta, n)$ distributed then there is texture present.

By the convergence property presented in equation (5), the $K A(\alpha, \beta, n)$ distribution is equivalent to a $\sqrt{\Gamma}(n, n / \beta)$ distribution when $x \rightarrow \infty$. Since the former case characterizes textured target and the latter characterizes smooth targets, an estimate of the parameter $x$ of the K-distribution (denoted by $\hat{x}$ ) may be used as a texture measure. If $\hat{x}$ is 'large' then the population is homogeneous; if $\hat{\alpha}$ is 'small' then the region is highly textured. The borderline between these two cases was found by Yanasse $e t$ al. (1993) to be when $\hat{x}$ has a value of approximately 10 . 
An estimate of the parameter $\%$. based on the substitution method, is given by the solution of the equation

$$
\sqrt{\frac{m_{2}}{\hat{\alpha} \hat{n}}} \frac{\Gamma(\hat{x}+1 / 2) \Gamma(\hat{n}+12)}{\Gamma(\hat{x}) \Gamma(\hat{n})}-m_{1}=0
$$

where $m_{j}=N^{-1} \sum_{i=1}^{N} z_{i}^{j}$ is the $j$-th sample moment based on the $N$ observations $\left(z_{i}\right)_{1 \leqslant i \leqslant s}$ and $n \hat{n}$ is an estimator of the equivalent number of looks.

In the case of the SAREX data from Tapajós, $n$ was estimated to be $5 \cdot 6$ (Yanasse et al. 1993) and equation (8) was solved by the Newton-Raphson method. In this analysis, the reciprocal of $\alpha$ is used as a texture measure as it can be compared more easily to other techniques.

\subsection{Coefficient of variation}

The coefficient of variation (CV) is defined as the ratio of the standard deviation to the mean and is often used to characterize the inhomogeneity of a population. It follows that

if $Z \sim K A(\alpha, \beta, n)$ then

$$
C V(Z)=\sqrt{\frac{\Gamma(x+1) \Gamma(x) \Gamma(n+1) \Gamma(n)}{\Gamma^{2}(x+1 / 2) \Gamma^{2}(n+1 / 2)}-1}
$$

and if $Z \sim \sqrt{\Gamma}(n, n / \beta)$ then

$$
C V(Z)=\sqrt{\frac{\Gamma(n+1) \Gamma(n)}{\Gamma^{2}(n+1,2)}-1}
$$

where $n$ is the equivalent number of looks.

There is a strong relationship between equations (8) and (9) since both are derived from the ratio between the second moment to the square of the first. The main difference between them is that the first is model based, and suitable only for $\mathrm{K}$-distributed observations, while the second is a signal-to-noise measure that can be applied to any set of data. Also. when $x \rightarrow x$ equations (9) and (10) coincide. Other estimation techniques can be seen in Oliver (1994).

The CV may be used as a measure of texture. Its usefulness lies in the fact that it is computationally very simple.

\subsection{Grey level co-occurrence matrix}

The use of the grey level co-occurrence matrix (GLCM) as a means of measuring texture in images has been well documented (Marceau et al. 1990, Kilpela and Heikkila 1990. Weszka et al. 1976). The GLCM describes the discrete joint probability function of the values of two pixels separated by a specified distance within an image segment. Haralick et al. (1973) proposed several measures that can be used to extract useful textural information from a GLCM.

Because the GLCM is calculated for a given pixel separation, it is sensitive to the scale and directionality of image texture. It also requires that the horizontal and vertical offsets of the two pixels be specified along with the size of image segment over which the GLCM should be constructed (the texture window). The texture within the virgin forest areas of the SAR imagery has an apparent scale of approximately eight georegistered pixels $(48 \mathrm{~m})$ and has no obvious directionality. The most 
appropriate GLCM texture measure, size of texture window and offsets were chosen by visually analysing their effect on the discrimination between virgin forest, regenerating forest and pasture areas. As a result, the contrast texture measure (a measure of the degree of spread of the GLCM (Haralick et al. 1973)), a window size of 17 by 17 pixels, a horizontal offset of zero and a vertical offset of seven pixels were implemented.

\section{Estimation of the age of regeneration}

A method of independently assessing the type of land cover was required so that the stage of regeneration could be compared to the measured texture values. This was achieved by analysing a sequence of Landsat Thematic Mapper (TM) images of the Tapajós region going back six years in time using a method similar to that of Lucas et al. (1993).

Images with less than 10 per cent cloud cover were acquired from the Brazilian Instituto Nacional de Pesquisas Espaciais (INPE) Cachoeira Paulista receiving and archiving station for each of the seven years, 1986 to 1992 . The predominant cloud cover associated with such tropical forest areas has prevented the acquisition of useful TM data before or since this sequence and suggests that this is a very fortunate run of optical imagery.

The TM imagery was co-registered to a UTM projection with the help of $1: 250000$ topographic maps. The geographical area was matched to that of the SAR imagery ( $24 \mathrm{~km}$ by $24 \mathrm{~km}$ ) but the TM data was resampled to a grid of $25 \mathrm{~m}$ by $25 \mathrm{~m}$ pixels making 960 by 960 pixels. A cloud and water mask was generated for each image with a 20 class $\mathrm{K}$-means unsupervised classification followed by assignment to the mask of those classes clearly associated with cloud and water bodies.

Broad land cover classes were clearly distinguishable within each image. The extent of pasture and cleared land was evident from the characteristically low nearinfrared (NIR: TM band 4) and middle infrared (MIR: TM band 5) but high red (TM band 3) reflectance. Likewise, the extent of new vegetation was clear from the high NIR and MIR reflectances but low red signal. Virgin forest was characteristically darker in the NIR and MIR bands than the new vegetation because of shadowing and absorption. Each image (with cloud and open water bodies masked out) was reclassified with a 24 class $\mathrm{K}$-means algorithm. The resulting classes were then assigned to one of these four categories (virgin forest, clear-cut/pasture, regeneration/ cultivation and unclassified/cloud) with reference to the original images.

The classifications of the sequence of annual images were logically combined to characterize the change in land use between 1986 and 1992 when the SAR imagery was acquired. Stages in this multi-temporal classification scheme are illustrated in figure 2. Clear cut areas in the multi-temporal age map arise simply from the clearcuts located in the 1992 image. Virgin forest in the age map arises from virgin forest classifications in each of the seven year image sequence. Regeneration is divided into three classes. If an area of the image was classified as regeneration throughout the image sequence then in the age map it was classified as $>6$ years (regeneration of greater than six years duration). If regeneration in 1992, 1991, 1990 and 1989 appeared to be virgin forest or clear-cut in 1986, 1987 or 1988 then in the age map it was classified as 4-6 years of regeneration. If regeneration in 1992 appeared to be virgin forest or clear-cut in 1989, 1990 or 1991 then in the age map it was classified as 1-3 years of regeneration. In other cases, such as the small number of illogical combinations caused by misclassified pixels (e.g., clear cut followed by virgin forest), 


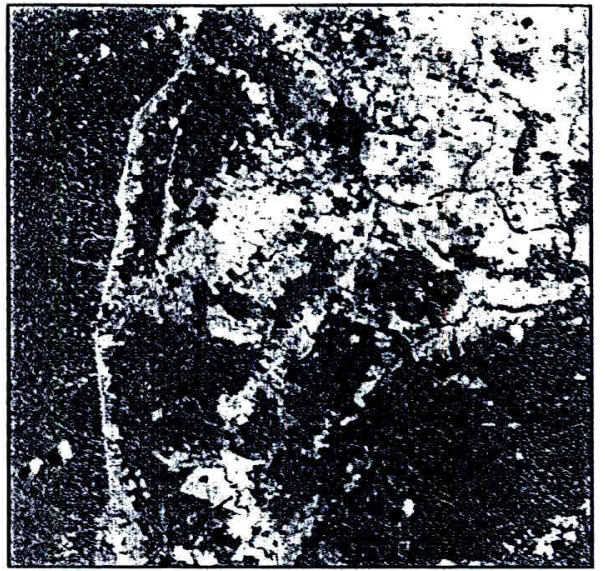

Landsat TM, 29th July 92; (R:4, G:5, B:3)

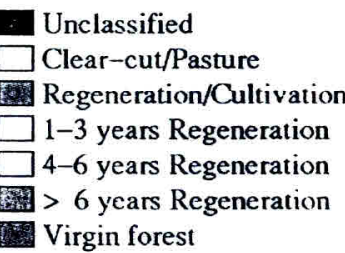

Key to Classes

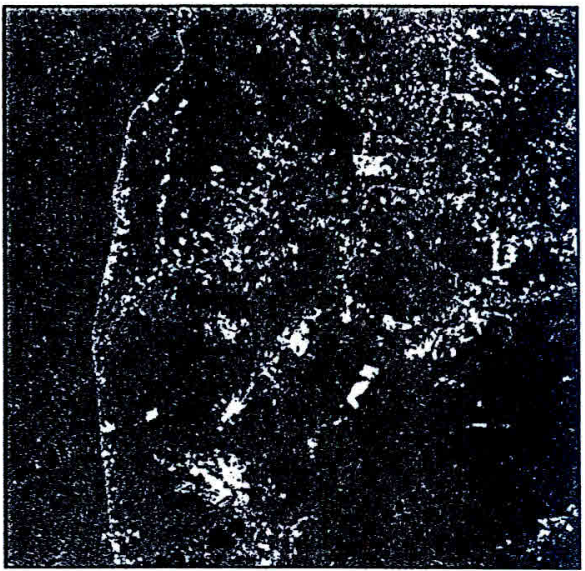

Classification into four classes

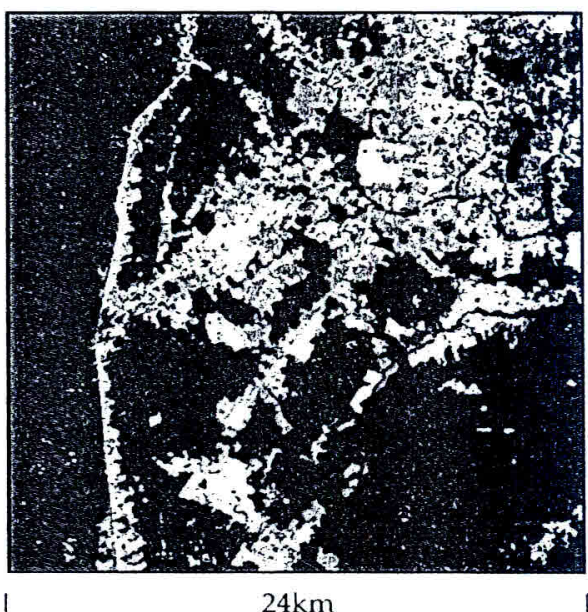

Seven-year multi-temporal classification

Figure 2. The Landsat TM multi-temporal classification. The multi-temporal age map is generated by classifying and logically combining a sequence of seven georegistered Landsat TM images. The top left image shows one of the TM scenes (the most recent) and the top right shows this scene classified into four classes (forest, regeneration, pasture and unknown/cloud). Seren such scenes acquired annually from 1986 to 1992 are combined to produce the age-map at the bottom right which gives an estimate of the extent of virgin forest and pasture and the duration of regrowth of the regenerating forest areas.

the pixel was treated as unclassified. Image class data missing in any year due to cloud cover was assumed to have not changed from the previous year. A summary of this scheme is given in table 1.

The resulting multi-temporal classification. or age map, was refined with a 5 by 5 mode filter to remove single-pixel features and each class was spatially eroded by one pixel to avoid edge contamination between classes. Each resulting individual polygon was given a unique label and age-class reference so that it could provide 
Table 1. A summary of the logical combination of annual TM classifications leading to the multi-temporal age map. Key: $\mathrm{C}=$ clear-cut/pasture, $\mathrm{R}=$ regeneration/cultivation, $\mathrm{F}=$ virgin forest, $-=$ any.

\begin{tabular}{|c|c|c|c|c|c|c|c|}
\hline 1986 & 1987 & 1988 & 1989 & 1990 & 1991 & 1992 & Age map \\
\hline - & - & - & - & - & - & $\mathrm{C}$ & Clear-cut \\
\hline - & - & - & - & - & $\mathrm{C}$ or $\mathrm{F}$ & $\mathbf{R}$ & \\
\hline - & - & - & - & $\mathrm{C}$ or $\mathrm{F}$ & $\mathrm{R}$ & $\mathbf{R}$ & $1-3$ years regeneration \\
\hline- & - & - & $\mathrm{C}$ or $\mathrm{F}$ & $\mathbf{R}$ & $\mathrm{R}$ & $\mathrm{R}$ & \\
\hline - & - & $C$ or $F$ & $\mathrm{R}$ & $\mathbf{R}$ & $\mathrm{R}$ & $\mathrm{R}$ & \\
\hline - & $\mathrm{C}$ or $\mathrm{F}$ & $\mathbf{R}$ & $\mathbf{R}$ & $\mathbf{R}$ & $\mathbf{R}$ & $\mathbf{R}$ & $4-6$ years regeneration \\
\hline $\mathrm{C}$ or $\mathrm{F}$ & $\mathbf{R}$ & $\mathbf{R}$ & $\mathrm{R}$ & $\mathbf{R}$ & $\mathrm{R}$ & $\mathbf{R}$ & \\
\hline $\mathrm{R}$ & $\mathbf{R}$ & $\mathrm{R}$ & $\mathrm{R}$ & $\mathrm{R}$ & $\mathbf{R}$ & $\mathrm{R}$ & $>6$ years regeneration \\
\hline $\mathrm{F}$ & $\mathrm{F}$ & $\mathrm{F}$ & $F$ & $\mathrm{~F}$ & $\mathrm{~F}$ & $\mathrm{~F}$ & Virgin forest \\
\hline
\end{tabular}

an example of a land parcel of a particular age of regeneration. This uniquely labelled age map was then resampled to the resolution of the georegistered SAR imagery ( $6 \mathrm{~m}$ by $6 \mathrm{~m}$ pixels) so that the texture measures could be compared to the age classification. Polygons of less than 1000 pixels in area were discarded to leave only those with a sufficient population over which to measure the texture.

The resulting age map was 4000 by 4000 pixels in size and contained 961 polygons, each of more than 1000 pixels in area, divided between the five age classes: clear-cut, 1-3 years. 4-6 years. $>6$ years and virgin forest. The texture, as measured by the three techniques discussed in $\$ 4$, was assessed over these polygon areas so that the relationship between age of regeneration and image texture could be investigated.

\section{Results and discussion}

Figures 3 and 4 show the $\mathrm{HH}$ polarized SAR imagery image amplitude and $\mathrm{CV}$ texture (in this case calculated within a 17 by 17 pixel sliding window) for the entire $24 \mathrm{~km}$ by $24 \mathrm{~km}$ area of interest. The $6 \mathrm{~km}$ by $6 \mathrm{~km}$ area within the black box is shown in more detail in figures 5 and 6 . Figure 5 is a colour composite of the $\mathrm{HH}$ image (in green) and the VV image (in magenta) while figure 6 shows the CV texture. Both images are overlaid in various colours by the boundaries of the polygons resulting from the multi-temporal TM classification. The image amplitude and three texture measures are calculated within each of these polygons and the results are quantified as a function of estimated regeneration age in figure 7 . The amplitude and texture values for the middle 67 per cent of polygons are plotted as individual points while the absolute maximum and minimum values for each class are indicated by the dashed lines. This reveals a large spread in values for each class but shows that the majority of this variability is due to only a few polygons.

\subsection{Image amplitude}

Despite the lack of absolute calibration of the SAR data, the relative $\mathrm{HH}$ to VV image amplitude vields interesting results. The mean backscatter amplitude for neither the $\mathrm{HH}$ nor the $\mathrm{VV}$ polarized images varies significantly between classes. Hence. as might be expected at C-band, the mean backscatter alone cannot be used even to discriminate between virgin forest and clear-cut areas. 


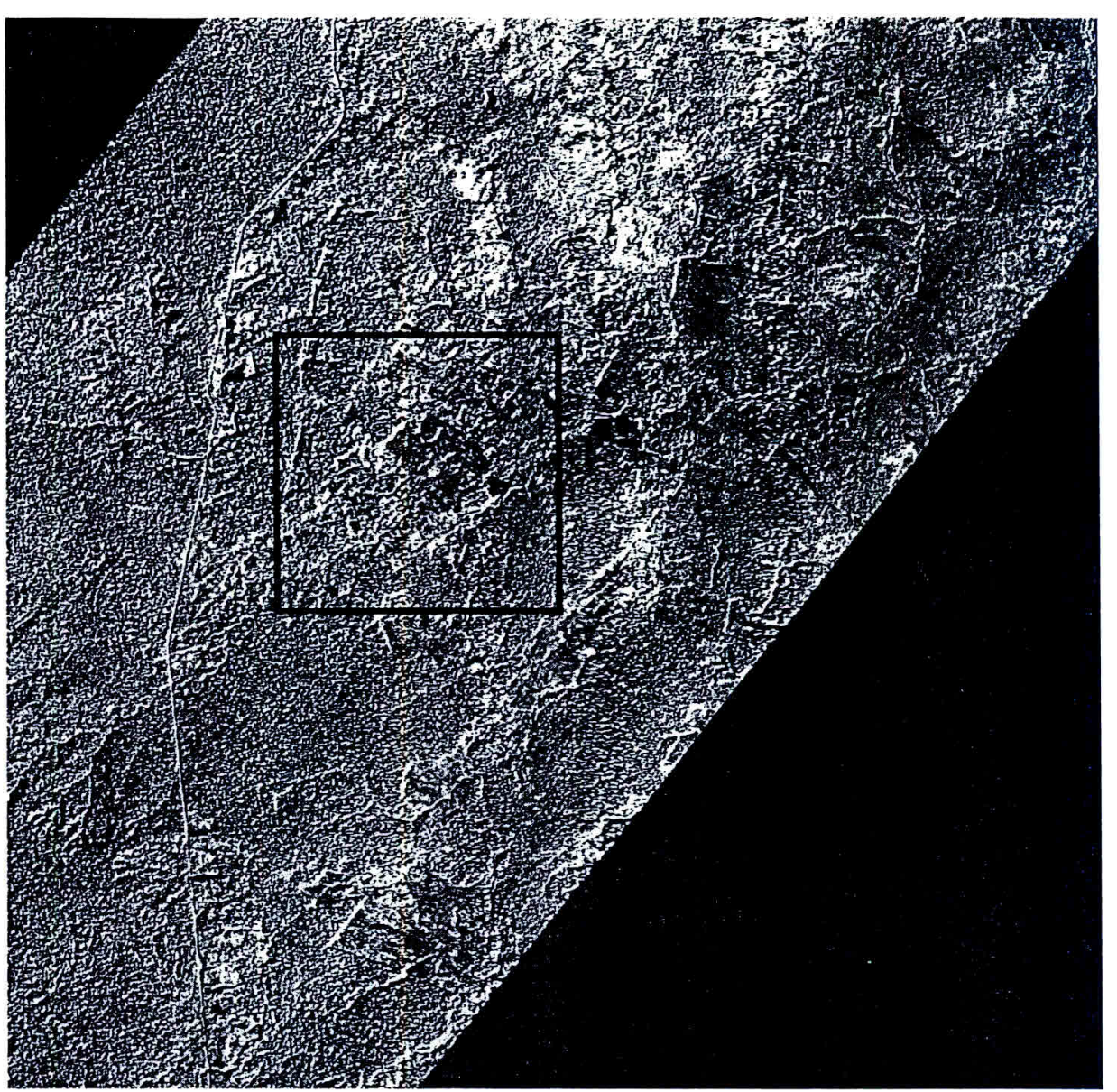

Figure 3. Overview of the SAR image from the Tapajos area of interest. The HH polarization image is shown and demonstrates the large textural variation across the area. The black box indicates the portion of the scene illustrated at full resolution in figure 5 .

The shape of the $\mathrm{HH}$ polarized amplitude response in figure 7 is similar to the $V V$ except from the clear-cut class for which the mean amplitude is relatively lower in the VV polarized image. This relative difference is indicated in figure 5 as a moremagenta colour in the vegetated areas (relatively higher VV) and a more-green colour in the clear-cut or pasture areas (relatively higher $\mathrm{HH}$ ). This may be due to the predominantly vertical orientation of scattering elements in the vegetated areas which scatter the vertically polarized radiation more strongly and has been previously noted by Shimabukuro et al. 119941.

\subsection{Image texture}

The images in figures 3 (overview) and 5 (full resolution) reveal the highly textured nature of the SAR data from this area. Three polygons have been labelled in figures 5 (SAR data) and Figure $6 / \mathrm{Cl}^{\prime}$ texture) to demonstrate the variation of texture with regeneration age. Polygon A represents virgin forest: the spatially coarse nature of 


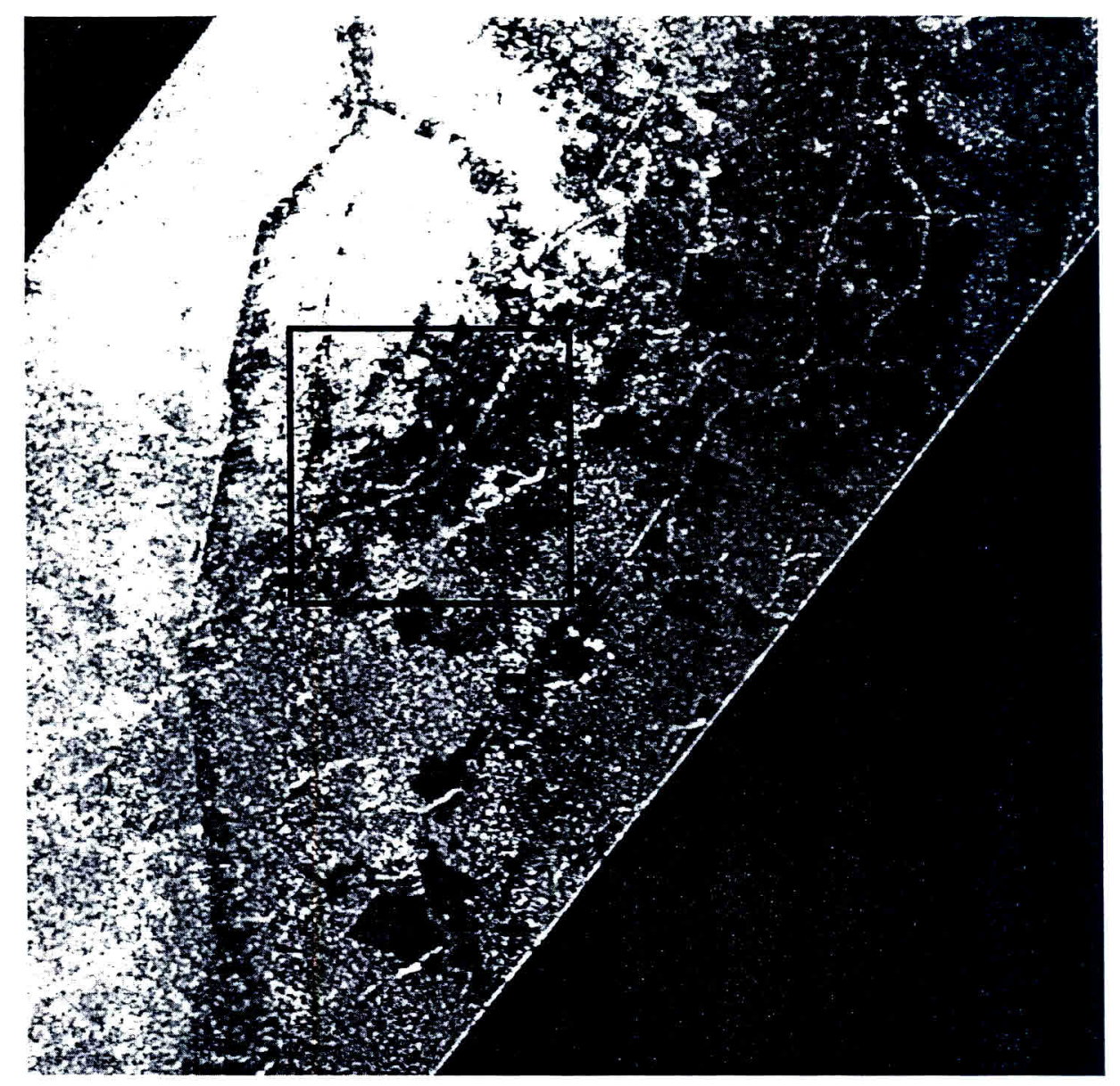

Figure 4. Overview of the texture image from the Tapajós area of interest. The coefficient of variation texture parameter has been calculated in a 17 by 17 pixel sliding window. The black box indicates the portion of the image illustrated at full resolution in figure 6 .

this texture is visible in figure 5 and figure 6 shows that the texture measure quantifies the texture as high (white). Polygon $B$ represents old regeneration ( $>6$ years) and the medium texture can be seen from both the imagery in figure 5 and the mid-grey colour in figure 6 . The surface roughness of the canopy in this polygon is visibly less than the virgin forest but more textured than other areas of the image. Polygon $\mathrm{C}$ is a clear-cut area; the imagery appears smooth and the texture image appears black over most of its area. Hence it would appear that at least three levels of texture discrimination are possible from this qualitative analysis of the imagery. All virgin forest areas appear to have similar visibly coarse texture, clear-cut areas appear to have very little texture and stages of regeneration exhibit texture that falls somewhere between these two extremes.

Three texture analysis techniques were employed in this study, the $\mathrm{CV}$, the GLCM contrast measure and the reciprocal of the K-distribution $\%$ parameter. The relationship between each of these texture measures and the estimated regeneration 

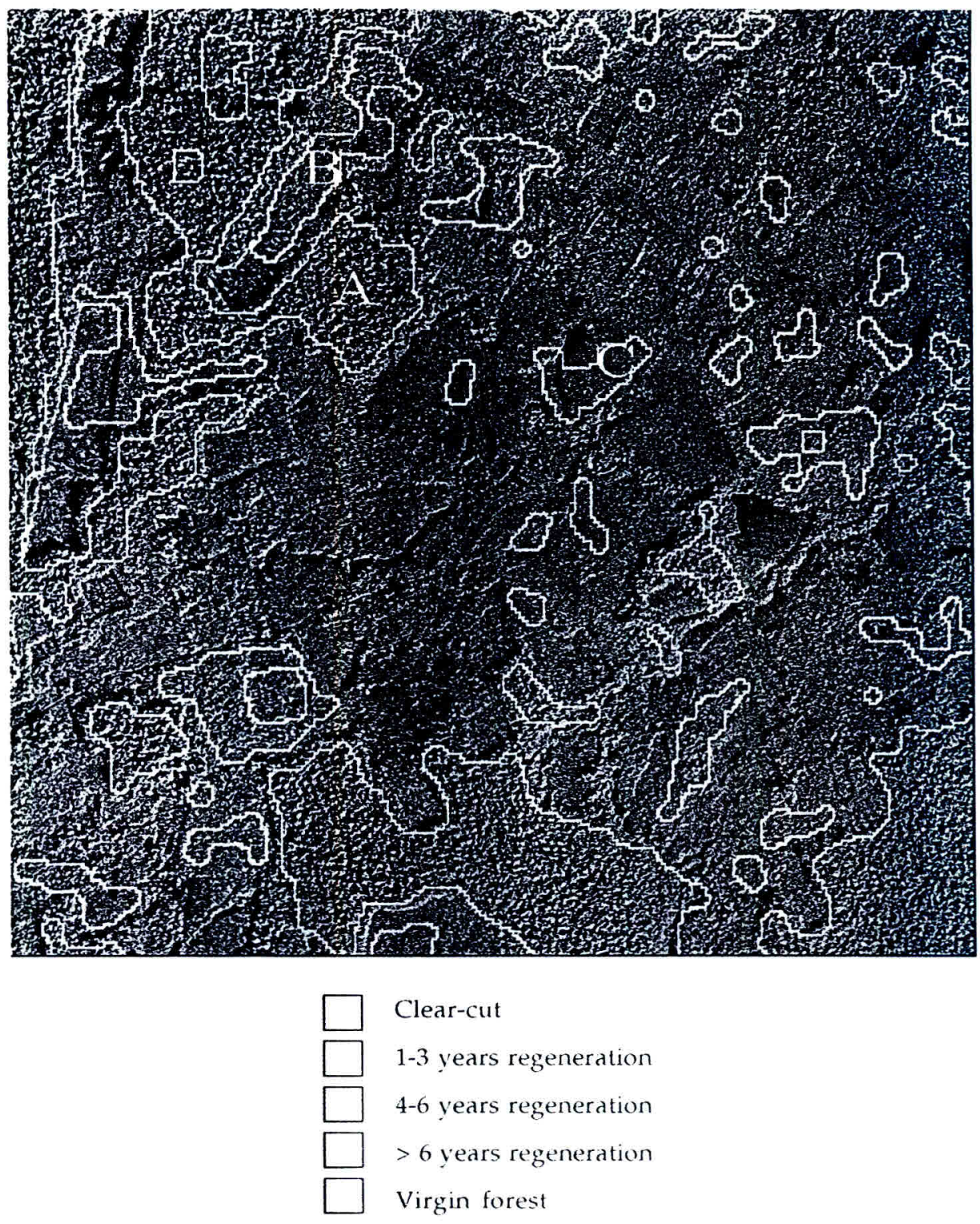

Kev to vector overlav colours

Figure 5. Colour composite of a small part of the SAR image (HH: green, VV: magenta). This image illustrates the variety of textures within areas of different estimated age of regeneration. The boundaries of the polygons resulting from the multi-temporal TM classificition are overlaid in various colours. The labelled polygons are discussed in the text.

age for all polvoons is shown in figure 7 . Each measure shows a much larger variation between different age classes than that shown by the SAR image amplitude alone and the shape of the responses are remarkably similar for all texture measures and 

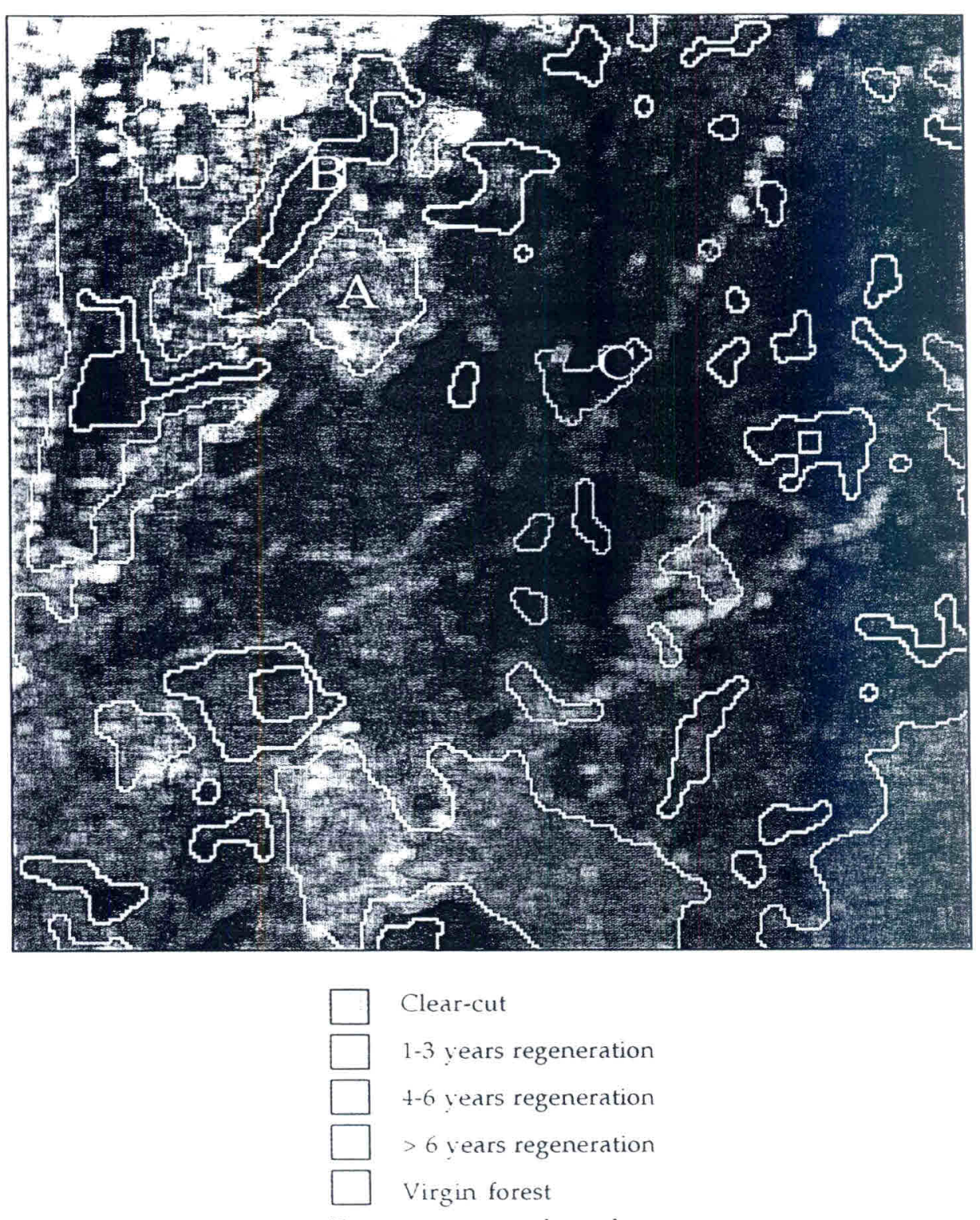

Key to vector overlay colours

Figure 6. A small part of the $\mathrm{Cr}$ texture image. The boundaries of the polygons resulting from the multi-temporal TN classification are overlaid in various colours. At least two levels of grey are visible within the disturbed part of the forest. The labelled polygons are discussed in the text

for both polarizations. This suggests that similar texture features are being detected by each technique and that the useful information in the texture is greater than that in the amplitude alone.

Despite the apparent ability of the texture measure to discriminate between at 

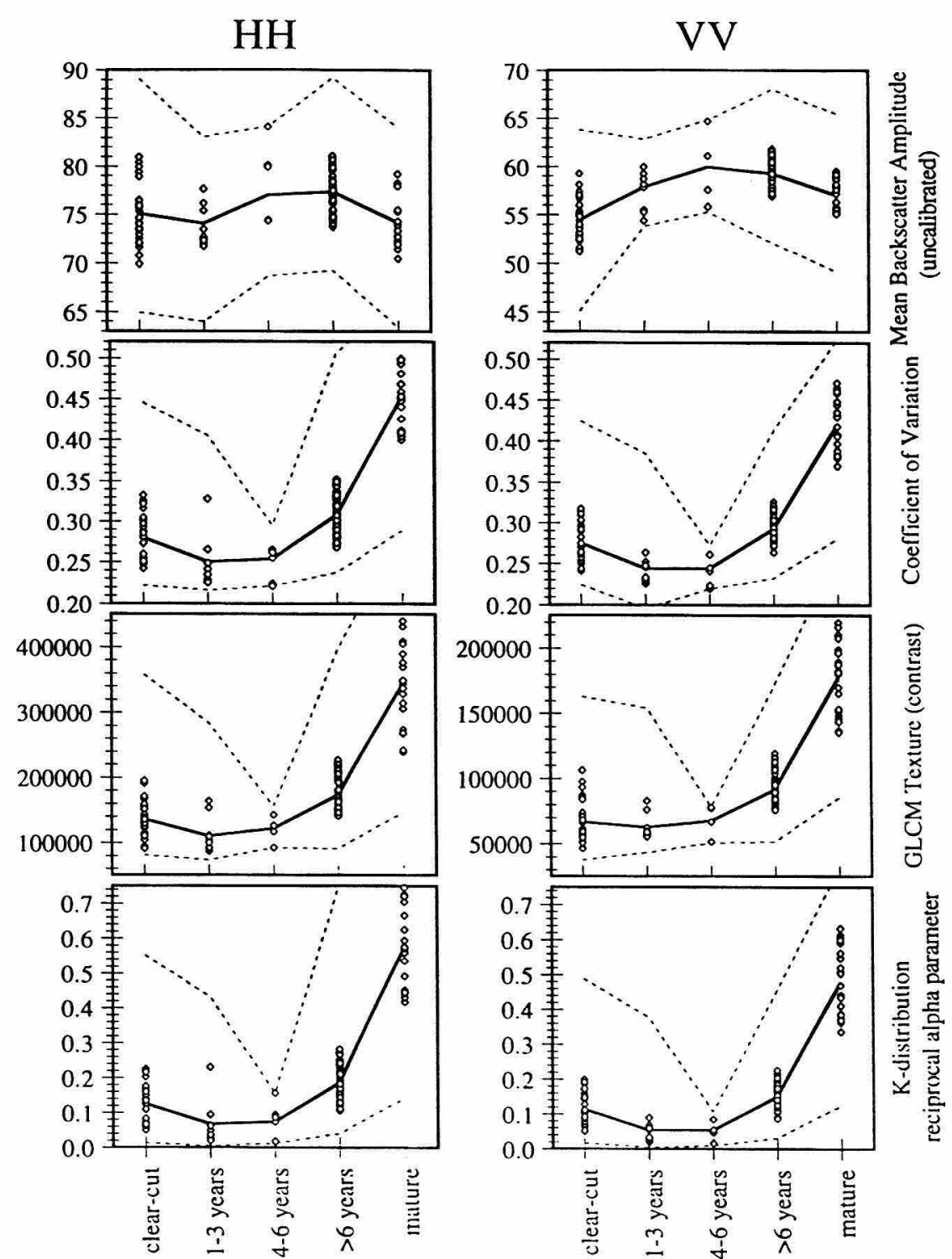

Figure 7. The relationship between estimated age of regeneration and the image parameters. The graphs show the relationship between the regrowth age, estimated from the multitemporal TM analysis, and the SAR amplitude and texture for each polarization and each texture measure. Each plotted point represents the average value within a single polygon of each class (after filtering and erosion). The middle $67 \%$ of polygons have been plotted and their means are indicated by the bold lines. The dashed lines represent the maximum and minimum value for all polygons within each class.

least three regeneration classes as illustrated by the texture image in figure 6 , the quantitative analysis in figure 7 suggests that the texture measures are capable of discriminating only between virgin forest and other classes. This inconsistency is most likely to be due to problems with the age map which is the only available 
Table 2. Coefficients of variation of each texture measure (CV, reciprocal $\alpha$ and GLCM) within each vegetation class and for each of the $\mathrm{HH}$ and VV polarized images.

\begin{tabular}{lcccccc}
\hline Class & $\begin{array}{c}\text { HH } \\
\text { CV }\end{array}$ & $\begin{array}{c}\text { VV } \\
\text { CV }\end{array}$ & $\begin{array}{c}\text { HH } \\
\text { 1/alpha }\end{array}$ & $\begin{array}{c}\text { VV } \\
\text { 1/alpha }\end{array}$ & $\begin{array}{c}\text { HH } \\
\text { GLCM }\end{array}$ & $\begin{array}{c}\text { VV } \\
\text { GLCM }\end{array}$ \\
\hline Clear-cut & 0.1042 & 0.0878 & 0.4719 & 0.4128 & 0.2161 & 0.2417 \\
1-3 years regeneration & 0.1258 & 0.0565 & 0.9072 & 0.5263 & 0.2430 & 0.1488 \\
4-6 years regeneration & 0.0867 & 0.0705 & 0.5764 & 0.5476 & 0.1909 & 0.1617 \\
$>$ 6 years regeneration & 0.0690 & 0.0513 & 0.2461 & 0.2286 & 0.1487 & 0.1283 \\
Virgin forest & 0.0719 & 0.0759 & 0.1808 & 0.1947 & 0.1704 & 0.1526
\end{tabular}

independent method of estimating the age of the regenerating forest. For example, the Landsat TM acquisitions may have been acquired during different parts of the local growing season giving rise to confusion between cultivated land and regrowing forest. More importantly, edge features in the images such as roads, rivers and abrupt changes in land cover, which have a more than average likelihood of being associated with clear-cut areas, may give rise to high image texture. These will be detected by the texture analysis-but not in the multi-temporal TM classification and may lead to anomalously high texture values. An example of this is shown in figures 5 and 6 . Area $C$ has been classified as clear-cut but the SAR image shows a river valley that dissects the plot and causes a high texture anomaly within an otherwise low textured region.

Although the three texture measures exhibit similar behaviours as a function of vegetation age class, it is possible to compare their precisions by examining the coefficient of variation of each texture measure for each age class. Table 2 shows the result of this analysis and indicates that the $\mathrm{CV}$ texture measure exhibits the least variability with respect to the mean, while the reciprocal of the K-distribution $x$ parameter exhibits the most. Hence the texture measure that would allow the best separation between age classes appears to be the $\mathrm{CV}$ which is also the least computationally complex measure to implement. However, the reciprocal of the K-distribution $\alpha$ parameter has the clearest interpretation with respect to the SAR image modelling as presented in equations ( 81 and (9). Its precision may be improved by considering related texture estimators such as those given by the use of normalized moments (Oliver 1994).

\section{Conclusions}

It is believed that, following disturbance by humans, the homogeneity of a regenerating tropical forest canopy decreases with time as regrowth species give way to emergent hardwood species. This study has demonstrated that the texture in high resolution C-band SAR images of such regenerating tropical forest at Tapajós in Brazil is affected by these changes during the regeneration process and that simple measures of image texture can be used to quantify these changes in texture.

A qualitative analysis of texture discrimination between different stages of regrowth suggests that at least three regeneration stages may be separated by simple texture measures. A quantitative analysis requires an independent method of verifying the duration of regrowth at each location within the image and this is provided by the analysis of a multi-temporal sequence of Landsat TM images which quantifies the regeneration age into fite classes. This quantitative analysis shows that image texture may be used to discriminate between mature forest and other classes of land 
cover where the image amplitude alone cannot be used to make the same distinction. Further discrimination between different stages of regrowth is not demonstrated despite the positive qualitative analysis. This is attributed partly to shortcomings with the multi-temporal Landsat TM age map which was not of sufficient resolution to detect features which might exhibit anomalously high texture such as roads and ravines.

The quantitative assessment of image texture using three different techniques, the coefficient of variation, the GLCM contrast and the reciprocal of the K-distribution $\alpha$ parameter has shown that image texture can yield an extra dimension from data that shows little variation in amplitude between different land cover classes. Of the three texture measures considered, the coefficient of variation exhibits the least variation within each vegetation age class and is therefore the most suitable for this data, as well as being the least computationally expensive.

\section{Acknowledgments}

We would like to acknowledge the Royal Society and the Academia Brasileira de Ciências for supporting a scientific exchange programme between INPE and BNSC. We are grateful for the SAR data which was provided through the joint ESA/CCRS SAREX campaign and for the sequence of Landsat TM imagery which was provided by INPE. This work was supported by the Natural Environment Research Council (NERC) and by INPE.

\section{References}

CAVEs, R. G., 1993, Automatic matching of features in synthetic aperture radar data to digital map data, PhD thesis, University of Sheffield, Sheffield, U.K.

Groom, G. B., Luckman, A. J., Baker, J. R., and Cordey, R. A., 1993, SAR image texture and spatial resolution for tropical forest mapping. Proceedings of the ESA SAREX-92 Workshop, ESA Headquarters (Paris: ESA), pp. 95-100.

Grover. K. D., and Quegan, S., 1995. ERS-1 observations and potential for use in tropical forest monitoring. Proceedings of IGARSS'95, Firenze, Italy (New York: I.E.E.E.), pp. $1210-1212$

Haralick, R. M., Shanmugam, K., and Dinstein, I., 1973. Textural features for image classification. I.E.E.E. Transactions on Systems, Man, and Cybernetics, 3, 610-621.

Hawkins, R. K., and Teany, L. D., 1993, SAREX 1992, data calibration. Proceedings of the ESA SAREX-92 Workshop, ESA Headquarters (Paris: ESA), pp. 41-53.

KILPELA, E., and HeikKILA, J., 1990, Comparison of some texture classifiers. ISPRS Symposium on Global and Environmental Monitoring, Victoria, B. C., Canada (Ann Arbor, MI: ERIM) pp. 333-339.

Lucas, R. M. Honzak, M, Foody G. M. Curran, P. J, and Corves, C., 1993 , Characterising tropical secondary forests using multi-temporal Landsat sensor imagery. International Journal of Remote Sensing, 14, 3016-3067.

Marceau, D. J., Howarth, P. J., Dubois, J. M., and Gratton, D. J., 1990, Evaluation of the grey-level co-occurrence matrix method for land-cover classification using SPOT imagery. I.E.E.E. Transactions on Geoscience and Remote Sensing, 28, 513-519.

Oliver, C. J., 1991, Information from SAR images. Journal of Physics D: Applied Physics, 24 1493-1514.

Oliver. C. J., 1994. Edge detection in SAR segmentation. Proceedings of the European Symposium on Satellite Remote Sensing: SAR Data Processing for Remote Sensing, (Rome: SPIE), vol. 2316, pp. 80-91.

QUEGAN, S., 1990, Interpolation and sampling in SAR images. I.E.E.E. Transactions on Geoscience and Remote Sensing. 28, 641-646.

Shimabukuro, Y. T., Lawrence. W. T.. and Ahern, F. J., 1994, Tropical vegetation analysis with Landsat Thematic Mapper and Canadian sunthetic aperture radar data 
Procedings of the European SImposium on Satellite Remote Sensing (Rome: SPIE), vol. 2314, pp. 255-260.

UHI., C.. 1987, Factors controlling succession following slash-and-burn agriculture in Amazonia. Journal of Ecology: 75, 377-407.

IAN DI:R SANIDIN, J. J., and HolkMiAx. D. H.. 1993. Identification of tropical forest cover types using X-and C-band airborne SAR data. Proceedings of the ESA SAREX-92 Workshop. ESA Headquarters (Paris: ESA), pp. 177-181.

WIST.KA. J. S., DYER, C. R., and Rosenfeld, A.. 1976, A comparative study of texture measures for terrain classification. I.E.E.E. Transactions on Sistems, Man. and Cybernetics, 6, 269-285.

Wooming, M. G., Zmuda. A. D.. and Attema, E.. 1994, An overview of SAREX-92 data acquisition and analysis of the tropical forest environment. Proceedings of the ESA SAREX-92 Workshop, ESA Headquarters (Paris: ESA), pp. 3-14.

YANASSE, C. C. F., Frerr. A. C., and Sant'ANNa, S. J. S., 1995. Stochastic distributions and the multiplicative model: relations, properties, estimators and applications to SAR image analysis. INPE, Sao Jose dos Campos (INPE-5360-NTC 318).

Yanasse, C. C. F., Frery, A. C., S.a.t Anna, S. J. S., Hernandez Filho, P. H., and Ditra, L. V., 1993, Statistical analvsis of SAREX data over the Tapajós. Brazil. Proceedings of the ESA SAREX-92 Workshop, ESA Headquarters (Paris: ESA). pp. 25-40. 